$\left(x_{k}<x_{k}^{\prime}\right)$. Then we obtain $\mathfrak{M}$ by removing from the plane of $z$ the two circular domains $|z|<\rho_{0}$ and $|z|>\rho$ and all annuli $x_{k}<|z|<x_{k}^{\prime}$, corresponding to the equations (14) with two different positive roots.

It is then a consequence of Walsh's theorem that the different (open) intervals $\left(x_{k}, x_{k}^{\prime}\right)$ have no points in common and lie in the interval $\left(\rho_{0}, \rho\right)$; moreover, if for two of these intervals $\left(x_{k}, x_{k}^{\prime}\right)$, $\left(x_{m}, x_{m}^{\prime}\right)$ we have $k<m$, then we have certainly $x_{k}^{\prime} \leqq x_{m}$, and it is easily seen that we have even $x_{k}^{\prime}<x_{m}$.

As Walsh remarks, his proof of Pellet's theorem remains valid also in the case of a power-series and of its roots inside the circle of convergence. It is hardly necessary to remark that our proof of Walsh's theorem also applies mutatis mutandis to a power series, if we only consider its roots within the circle of convergence.

UNIVERSITY OF BASEL

\title{
SOME EXCEPTIONAL VALUES OF THE LIMIT OF THE RATIO OF ARC TO CHORD
}

\section{RICHARD COHN}

It was observed by $\mathrm{E}$. Kasner ${ }^{1}$ that in the complex euclidean plane the limiting value of the ratio of the arc of a curve to its chord, while one end point of the arc is fixed, and the other approaches it along the curve, is not always unity; but assumes for analytic curves tangent to a minimal line, a sequence of real values, $.94 \cdots, .86 \cdots, .80, \cdots$. These values are functions of the order of contact only, and approach zero as the latter increases. In this note we shall describe two similar situations which occur in real spaces.

The problem in the case of the $K$ plane $^{2}$ has been worked out in Professor Kasner's Seminar in Geometry. ${ }^{3}$ In this plane the length of the curve $y=f(x)$ passing between points of abscissae $x_{1}, x_{2}$, in that order, is given by

$$
l=\int_{x_{1}}^{x_{2}}\left(\frac{d y}{d x}\right)^{-1} d x .
$$

${ }^{1}$ E. Kasner, The ratio of the arc to the chord of an analytic curve need not approach unity, this Bulletin, vol. 21 (1914), pp. 524-531. Similar questions for three dimensions are discussed in E. Kasner, Complex geometry and relativity, theory of the "rac" curvature, Proceedings of the National Academy of Sciences, 1932, p. 267.

${ }^{2}$ Kasner, Trihornometry, a new chapter of conformal geometry, Proceedings of the National Academy of Sciences, vol. 23, p. 346.

${ }^{3} \mathrm{R}$. Coleman, S. Jablon and D. Mittleman obtained the results for the $K$ plane given below. 
The distance from the point $\left(x_{1}, y_{1}\right)$ to the point $\left(x_{2}, y_{2}\right)$ is defined as the length of the straight line passing from the first of these points to the second and is therefore given by the equation

$$
c=\frac{\left(x_{2}-x_{1}\right)^{2}}{y_{2}-y_{1}} .
$$

We see that lines parallel to the $x$-axis have infinite length while those parallel to the $y$-axis are the minimal lines or lines of zero length. We shall now investigate the ratio of the length of an analytic arc to the distance between its end points, both length and distance being used in the sense just defined.

The most interesting cases will be those of curves tangent to a minimal line. We will obviously lose nothing by choosing the origin for the point of contact. We will write the equation of our curve in the form $x=a_{n} y^{n}+a_{n+1} y^{n+1}+\cdots, a_{n} \neq 0$. Evidently this includes the normal as well as the exceptional situations; for when $n=1$ the curve cuts the minimal line, for greater values of $n$ it is tangent to it.

We may write for the length of the curve from the origin to the point $(X, Y)$ the equation $l=\int_{0}^{Y}(d x / d y)^{2} d y$. This is obtained easily from the expression (1) for the length. Since $d x / d y=n a_{n} y^{n-1}+\cdots$, this becomes

$$
\begin{aligned}
& l=\int_{0}^{Y}\left(n a_{n} y^{n-1}+\cdots\right)^{2} d y=\int_{0}^{Y}\left(n^{2} a_{n}^{2} y^{2 n-2}+\cdots\right) d y, \\
& l=\frac{n^{2}}{2 n-1} a_{n}^{2} Y^{2 n-1}+\cdots .
\end{aligned}
$$

The distance from the origin to the point $(X, Y)$ is seen to be

$$
c=\frac{X^{2}}{Y}=\frac{\left(a_{n} Y^{n}+\cdots\right)^{2}}{Y}=a_{n}^{2} Y^{2 n-1}+\cdots .
$$

The first terms of these expansions do not vanish, so that as $Y$ approaches zero, $l / c$ approaches their quotient $n^{2} /(2 n-1)$ as limit. For $n=1$ this is unity, but for curves tangent to the minimal line this limit assumes a sequence of values $4 / 3,9 / 5,25 / 4, \cdots$, increasing towards infinity with the order of contact.

There remains the case of curves tangent to a line of infinite length. These must be written in the form $y=a_{n} x^{n}+\cdots, a_{n} \neq 0, n>1$. A calculation similar to the preceding one shows that such curves have infinite arc length measured from the point of tangency to any other point, while the corresponding chords are finite. 
In the affine differential geometry of the plane which has been developed by Blaschke ${ }^{4}$ and others we work with elements consisting of a point and a slope. For a curve to pass from the element consisting of the point $\left(x_{1}, y_{1}\right)$ and the slope $y_{1}^{\prime}$ to the element consisting of the point $\left(x_{2}, y_{2}\right)$ and the slope $y_{2}^{\prime}$ it must pass through each point and have at each the corresponding slope. If $y=f(x)$ is the equation of such a curve, the affine length of the arc between these elements is defined by

$$
l=\int_{x_{1}}^{x_{2}}\left(\frac{d^{2} y}{d x^{2}}\right)^{1 / 3} d x .
$$

This integral is invariant under an area-preserving affine transformation. With the given end-conditions it becomes stationary when the arc is a parabola. Its value in this case is a relative maximum and is called the affine distance between the elements.

I have carried over the problem to this case by considering the limit of the ratio of the affine length of an arc to the affine distance between the elements tangent to the arc at its end points, as one end point is fixed and the other approaches it along the curve.

It will be observed that all straight lines have zero affine length, and it is to be expected, by analogy with the preceding cases, that unusual values of the limit will occur for those curves and only those which have high order of contact with a straight line. That this is indeed the case is shown in the following calculations.

By means of a suitable translation and rotation, which cannot affect the quantities under investigation, we write any analytic curve in the form $y=a_{n} x^{n}+a_{n+1} x^{n+1}+\cdots, a_{n} \neq 0, n \geqq 2$. The affine length of this curve from the element at the origin with slope zero to the element tangent to the curve at the point $(X, Y)$ is found by means of (5) to be given by

$$
\begin{aligned}
l & =\int_{0}^{X}\left(\frac{d^{2} y}{d x^{2}}\right)^{1 / 3} d x=\int_{0}^{x}\left(n(n-1) a_{n} x^{n-2}+\cdots\right)^{1 / 3} d x, \\
\text { (6) } \quad l & =\int_{0}^{x}\left(\left[n(n-1) a_{n}\right]^{1 / 3} x^{(n-2) / 3}+\cdots\right) d x, \\
l & =\frac{\left[n(n-1) a_{n}\right]^{1 / 3}}{(n+1) / 3} X^{(n+1) / 3}+\cdots
\end{aligned}
$$

${ }^{4}$ W. Blaschke, Differentialgeometrie, vol. 2, chap. 1. (5) and (7) below are easily obtained from the equations given by Blaschke in vector notation. 
The general formula for the affine distance between the elements consisting of the points $\left(x_{1}, y_{1}\right),\left(x_{2}, y_{2}\right)$ with the slopes $y_{1}^{\prime}$ and $y_{2}^{\prime}$ respectively is

(7) $c=\left[\frac{4\left[\left(y_{2}-y_{1}\right)-y_{1}^{\prime}\left(x_{2}-x_{1}\right)\right]\left[y_{2}^{\prime}\left(x_{2}-x_{1}\right)-\left(y_{2}-y_{1}\right)\right]}{y_{2}^{\prime}-y_{1}^{\prime}}\right]^{1 / 3}$.

In the case under consideration this becomes

$$
\begin{aligned}
c & \left.=\left[\frac{4 Y}{Y^{\prime}}\left(X Y^{\prime}-Y\right)\right]^{1 / 3}, \quad Y^{\prime}=\frac{d y}{d x}\right]_{x=X}=n a_{n} X^{n-1}+\cdots \\
c & =\left[\frac{\left(4 a_{n} X^{n}+\cdots\right)\left(\left[n a_{n} X^{n}+\cdots\right]-\left[a_{n} X^{n}+\cdots\right]\right)}{n a_{n} X^{n-1}+\cdots}\right]^{1 / 3}, \\
c & =\left[\frac{4(n-1) a_{n}}{n}\right]^{1 / 3} X^{(n+1) / 3}+\cdots
\end{aligned}
$$

We observe that the first terms of the expansions for $l$ and $c$ do not vanish. Consequently as $X$ approaches zero the ratio of these quantities approaches

$$
\frac{3}{n+1}\left[\frac{n^{2}}{4}\right]^{1 / 3}
$$

Thus the limiting value of the ratio of affine arc to affine chord will be unity for the case $n=2$, but for curves with a higher order of contact with a straight line it will assume a sequence of values $.98 \cdots, .95 \cdots$, $.92 \cdots, \cdots$, dependent only on the order of contact and decreasing towards zero as the latter increases.

We have thus seen that there is a close agreement in the behavior of the limit of the ratio of the arc and chord of an analytic curve in the complex euclidean, the Kasner and the affine plane. In each case this quantity is unity for most curves, but may also have one of a denumerable set of other values. These values occur for curves with higher than the least possible order of contact with a minimal line, and depend only on that order of contact.

Columbia University 